\title{
Three-dimensional molecular dynamics simulations of void coalescence during dynamic fracture of ductile metals
}

\author{
E. T. Seppälä* J. Belak $\dagger$ and R. E. Rudd \\ Lawrence Livermore National Laboratory, Condensed Matter Physics Division, L-045, Livermore, CA 94551, USA
}

(Dated: October 23, 2018)

\begin{abstract}
Void coalescence and interaction in dynamic fracture of ductile metals have been investigated using three-dimensional strain-controlled multi-million atom molecular dynamics simulations of copper. The correlated growth of two voids during the coalescence process leading to fracture is investigated, both in terms of its onset and the ensuing dynamical interactions. Void interactions are quantified through the rate of reduction of the distance between the voids, through the correlated directional growth of the voids, and through correlated shape evolution of the voids. The critical inter-void ligament distance marking the onset of coalescence is shown to be approximately one void radius based on the quantification measurements used, independent of the initial separation distance between the voids and the strain-rate of the expansion of the system. The interaction of the voids is not reflected in the volumetric asymptotic growth rate of the voids, as demonstrated here. Finally, the practice of using a single void and periodic boundary conditions to study coalescence is examined critically and shown to produce results markedly different than the coalescence of a pair of isolated voids.
\end{abstract}

PACS numbers: 61.72.Qq, 62.20.Mk, 62.20.Fe, 61.72.Lk

\section{INTRODUCTION}

The fracture of ductile metals at high strain rates has been understood at the microscopic level as a process of


voids nucleate at the weak points in the material such as inclusions and/or grain boundary junctions. Once nucleated, the voids grow under the tensile stress, driven by the reduction in elastic energy. Eventually, the voids grow sufficiently large that they interact with each other, in some cases link through localized shear, coalesce into larger voids, and finally form the fracture surface ${ }^{3.4}$ Considerable experimental and theoretical work has gone into the development of our understanding of fracture. The fracture process has been modeled at various levels, but most of the work involving the simulation of the activity of individual voids has concentrated on the void growth and its relationship to the plastic deformation of the surrounding material, including effects such as the localization of this shear deformation. Relatively little work has gone into the explicit modeling of void coalescence, and both the understanding of the physics of this process and the knowledge of how it should be implemented robustly in continuum fracture codes remain open issues. The point at which voids begin to coalesce during dynamic fracture is of considerable interest because complete fracture of the material typically ensues rapidly thereafter. As new experimental techniques have constrained the void growth models ever more stringently $\underline{\underline{5}}$ a real need for a well developed theory of coalescence has arisen.

Computationally void growth has been studied extensively at the continuum level, 6.7.8.9 also in dynamic, high strain-rate, conditions $\stackrel{10.11}{=}$ Recently we have studied void growth at the atomistic level under high strain-rate expansion, motivated by spallation experiments 12.13 .14 .15 .16 The atomistic studies demonstrate that voids grow by emitting dislocations that carry away the material, platelets of atoms, from the void and are responsible for the plastic deformations needed to accommodate significant void growth. There are also many recent studies of fracture in ductile metals with several holes or voids $\frac{17,18,19,20.21}{}$ While these studies model the void growth explicitly with fairly sophisticated models of plasticity in many cases, they typically simplify the coalescence process to instantaneous unification of the voids when some threshold is reached, such as once the voids grow to within one diameter of each other. There are also studies that investigate the competition between void by void growth versus multiple void interaction in crack propagation ${ }^{22}$ Several earlier continuum studies 23.24 .25 and the one atomistic study known to us ${ }^{26}$ of the coalescence process have been typically conducted in effectively two dimensional and highly symmetric systems.

This Article covers in detail a study of the onset of void coalescence. The first results of this study have been presented in a Letter ${ }^{27}$ Here we provide a more complete presentation of the results of our study of void coalescence. In addition to a more detailed description of the results presented in the Letter, we describe different measures of void interactions such as shape changes induced by a neighboring void, additional analysis of the behavior of the system including stress-strain curves and void volume curves, and a new analysis of how the coalescence of isolated pairs of voids differs from that of voids in highly symmetric periodic arrays.

In particular the goal of this Article is to quantify the point at which coalescence begins, as measured by a critical inter-void ligament distance (ILD), and examine the mechanisms involved in the transition from independent void growth to coalescence. There are several ways in which two voids can interact. In the case of pure impingement, the voids only interact when they grow to the point that they intersect and join into a single void. In reality, the voids interact before they intersect. Their 
range of interaction is extended due to their elastic and plastic fields. Each void generates an elastic strain field of the form generally associated with centers of dilatation $\stackrel{28}{\underline{2}}$ The shear stress decreases with the distance from the void like $r^{-3}$. For voids sufficiently close each void's growth rate is altered by the stress field of the proximal void. The modification of the elastic field can affect the initiation of plasticity, as well as the subsequent development of the plastic zone around the voids. The voids may interact through their plastic fields, too, in which case the fields may give rise to an increased hardening rate in a localized region or to thermal softening and shear localization. An argument due to Brown and Embury for a transition to shear deformation based on simple geometrical considerations suggests that the critical inter-void ligament distance, $\mathrm{ILD}_{c}$, should be equal to one diameter of a void ${ }^{29}$ that is, when the surfaces of a pair of voids are separated by one void diameter, they transition from independent void growth to coalescence. It is at this point, they argue, that the dominant void process switches from the radial plastic flow around isolated growing voids to a large-scale shear deformation allowing the rapid coalescence of the pair of voids. However, more recent two-dimensional studies suggest that for distances between voids as large as six diameters the void growth rate is enhanced 30

The use of atomistic techniques permits the analysis of the contributions of these competing mechanisms to the onset of void coalescence, as we describe in this Article. We demonstrate the existence of, and compute, the critical inter-void ligament distance $\mathrm{ILD}_{c}$ by starting with two voids well separated from each other and detecting the point at which correlated growth begins, marked both by the accelerated rate at which the two void surfaces approach each other and by biased growth causing the voids to start to extend toward each other. These changes give an indication of the onset of the coalescence process, and it tests the argument by Brown and Embury 29 We also test the setup by Horstemeyer et al ${ }^{30}$ by varying the initial distances between the voids and measuring the asymptotic growth rate of the voids. The initial void-to-void distance below which the growth-rate is enhanced should give another candidate for the critical distance and measure it in a volumetric sense. It should be noted, however, that the three-dimensional void coalescence studied here with molecular dynamics, as indeed any $3 \mathrm{D}$ coalescence of roughly spherical voids, does not admit the two-dimensional shear mechanism proposed by Brown and Embury in its simplest form, and therefore these different analyses are not fully comparable. Also Horstemeyer et al. used a more symmetric setup than the simulations covered in this Article.

The Article is organized as follows. The simulation method and the performed computations are introduced in Section III The basic mechanism, dislocation driven void growth, is demonstrated and the key reference parameter, mean linear void size, is introduced in Section IIII The interaction between voids are studied in

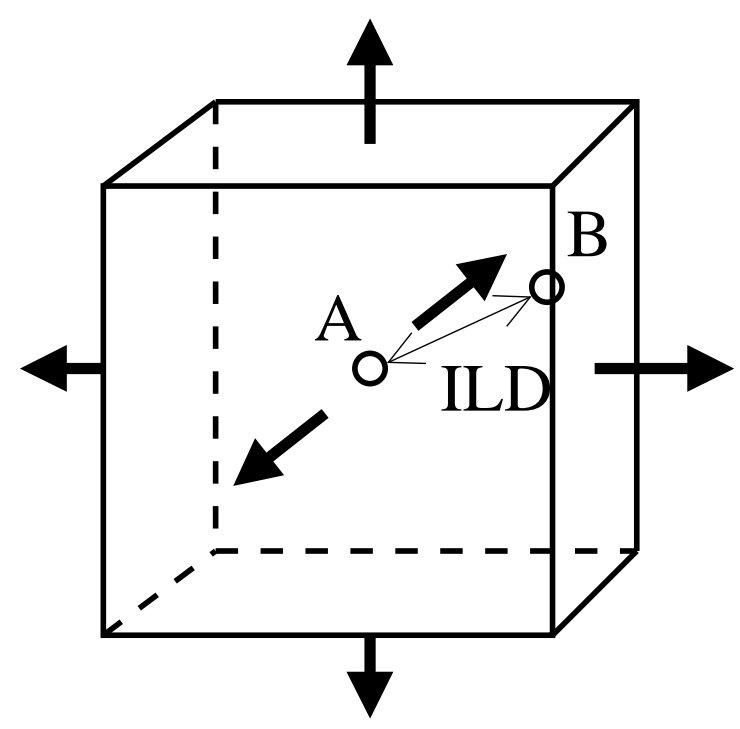

FIG. 1: A sketch of the simulation configuration. The simulation box includes $120 \times 120 \times 120$ FCC unit cells with periodic boundary conditions for a total of 6912000 atoms initially. From this two equal size voids are created by removing approximately 3620 atoms for each void. The thin arrow shows the inter-void ligament distance (ILD), the shortest surfaceto-surface distance between the voids, identified as A and B. The initial ILD ranges between one-half and five times the initial void radius. The bold arrows denote triaxial, hydrostatic, expansion of the box. The strain-controlled expansion is applied with constant strain-rates of $\dot{\varepsilon}=10^{8} / \mathrm{sec}$ and $10^{9} / \mathrm{sec}$.

TABLE I: The initial position for void B relative to void $\mathrm{A}$ and the center-to-center distances for various initial intervoid (surface-to-surface) ligament distances $\mathrm{ILD}_{0}$ used in this study.

\begin{tabular}{ccc}
\hline \hline $\mathrm{ILD}_{0}$ & $\begin{array}{c}\text { position of void B } \\
\text { relative to void A }\end{array}$ & $\begin{array}{c}\text { separation between the } \\
\text { centers of voids A and B }\end{array}$ \\
\hline 0.50 & {$[0.1334,0.0622,0.0290] L$} & $0.150 L=6.510 \mathrm{~nm}$ \\
1.00 & {$[0.1778,0.0829,0.0387] L$} & $0.200 L=8.680 \mathrm{~nm}$ \\
1.20 & {$[0.1956,0.0912,0.0426] L$} & $0.220 L=9.548 \mathrm{~nm}$ \\
1.50 & {$[0.2223,0.1037,0.0483] L$} & $0.250 L=10.85 \mathrm{~nm}$ \\
1.81 & {$[0.2500,0.1166,0.0544] L$} & $0.281 L=12.20 \mathrm{~nm}$ \\
4.62 & {$[0.5000,0.2332,0.1087] L$} & $0.562 L=24.40 \mathrm{~nm}$ \\
\hline \hline
\end{tabular}

Section IV Section IV A uses two different distance measurements to study the interaction; Section IVB introduces a shape parameter for the purpose. Finally the volume effects of the void growth are studied in Section $\nabla$ two separate voids are studied in Section $\nabla A$ and one void and the interaction with its periodic image are studied in Section $\mathrm{VB}$. The paper ends with conclusions, Section VI 


\section{METHOD AND SIMULATIONS}

We have performed a series of large-scale classical molecular dynamics (MD) simulations ${ }^{31}$ in single crystal face-centered cubic (FCC) systems using an empirical embedded-atom model (EAM) potential for copper 32.33 The three dimensional (3D) simulation box consists of $120 \times 120 \times 1204$-atom FCC cells with periodic boundary conditions for a total of 6912000 atoms, in most cases. In Section $\mathrm{VB}$ where results from smaller system size simulations are presented, the details of the sizes are introduced. For the nearly 7 million atom simulations the MD code was parallelized using spatial domain decomposition and run in a massively parallel computer using from 64 to 256 processors.

In the simulations the system is initially equilibrated using a thermostat ${ }^{34}$ at room temperature, $T=300 \mathrm{~K}$, and a constant volume $L^{3}$ (with $L=43.3 \mathrm{~nm}$ ) chosen to give ambient pressure, $P \simeq 0 \mathrm{MPa}$. Once the system has reached equilibrium, atoms are removed to create two spherical voids in the system with radius $r_{0}=0.05 L=2.17 \mathrm{~nm}$ : one centered in the box and the other located a distance $\operatorname{ILD}_{0}+2 r_{0}$ away in the direction $\hat{u}=[0.8892054,0.41464327,0.19335135]$ from the first void. We refer to these as void A and void B, respectively, see Fig. 1] $\mathrm{ILD}_{0}$, the initial inter-void ligament distance, is the closest surface-to-surface distance between the voids 35 and it is varied here, but the relative orientation of the voids is kept fixed. For $\mathrm{ILD}_{0}$ we have used the values: $1.00,1.20,1.50,1.81$, and in some cases 0.50 and 4.62 . The unit for the ILD is the void diameter, $d=2 r$. The positions for the center of the void B and the distances between the voids are listed in Table Initially, the voids are equal in size, with approximately 3620 atoms removed for each. This removal of atoms can be interpreted as an instantaneous debonding of two infinitely weakly bound inclusions.

Once the voids are formed, the thermostat is turned off, and dilatational strain is applied uniformly at a constant strain-rate $\dot{\varepsilon}$. The strain-controlled simulations 12 are carried out using the scaled coordinate formulation typically employed in the constant pressure method due to Parrinello and Rahman ${ }^{36}$ Use of scaled coordinates prevents the spurious generation of elastic waves at the box boundaries. In this method the positions of the atoms are stored using rescaled coordinates between $[0,1)$. When calculating the forces and new positions of the atoms their coordinates are multiplied by a diagonal scaling matrix $\mathcal{H}=\left\{L_{x}, L_{y}, L_{z}\right\}$. This scaling matrix is updated each time step, when the expansion is applied, by multiplying the initial matrix $\mathcal{H}_{0}=\{L, L, L\}$ with the sum of the unit matrix and the strain matrix $\mathcal{E}=t \dot{\mathcal{E}}$, $\mathcal{H}(t)=\mathcal{H}_{0}(\mathbb{I}+t \dot{\mathcal{E}})$. Applied strain-rates of $\dot{\varepsilon}=10^{8} / \mathrm{sec}$ and $10^{9} /$ sec have been used with perfectly triaxial, or hydrostatic, expansion, $\dot{\mathcal{E}}=\left\{\dot{\varepsilon}_{x}, \dot{\varepsilon}_{y}, \dot{\varepsilon}_{z}\right\}=\{\dot{\varepsilon}, \dot{\varepsilon}, \dot{\varepsilon}\}$. Thus $L_{x}(t)=L_{y}(t)=L_{z}(t)=L(t)=V^{1 / 3}$, where $V$ is the volume of the box. A time step of $6.7 \mathrm{fs}$ was used. More details of the simulation method can be found in Ref. 16,

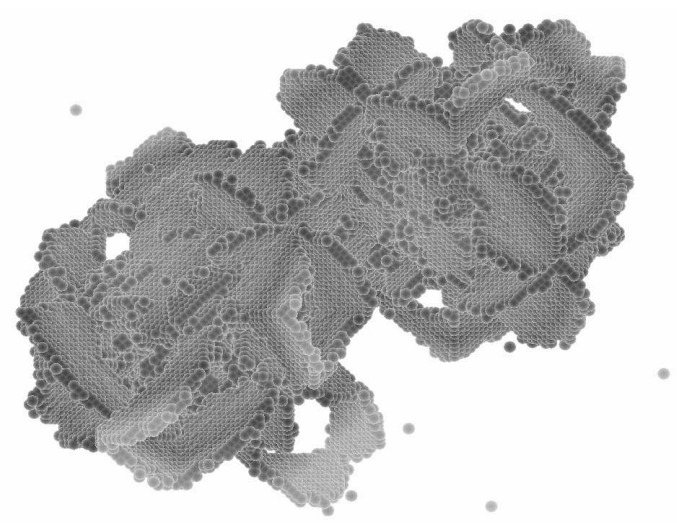

FIG. 2: (Gray-scale) A three dimensional snapshot of the two voids with the prismatic dislocation loops forming from the voids. Only those atoms belonging to the void surfaces or to dislocation cores, stacking faults or other defects are shown (and a small number of extraneous atoms due to thermal fluctuations). The stacking fault ribbons are the broad plates between leading and trailing partial dislocations. The snapshot is from the simulation with the initial inter-void ligament distance $\mathrm{ILD}_{0}=1.81$ diameters, and the strain-rate $\dot{\varepsilon}=10^{9} /$ sec. The strain at the snapshot is $\varepsilon=2.93 \%$.

including analysis of growth of a single void of the same initial radius $r_{0}$ in non-triaxial expansion.

\section{DISLOCATIONS AND VOID GROWTH}

Let us start reviewing the simulation results by looking at some figures to visualize growth of the voids. While some void growth takes place through elastic stretching in the initial phases of the box expansion, significant void growth and void-void interaction take place only once plastic deformation has begun. The important role of plasticity leads us to consider in some detail how dislocations are generated and the effect of dislocation dynamics on void coalescence, which are the topics of this Section.

In Fig. 2 a three-dimensional snapshot of the voids is shown from a simulation with the initial inter-void ligament distance $\mathrm{ILD}_{0}=1.81$ diameters and the strain-rate $\dot{\varepsilon}=10^{9} / \mathrm{sec}$. In the plot only the atoms at crystallographic defects such as void surfaces, dislocation cores, and stacking faults are shown. The decision of which atoms to plot is based on a geometrical criterion, a finitetemperature generalization of the centrosymmetry deviation. ${ }^{15.37}$ The power of this method of selecting atoms based on the centrosymmetry deviation is that the visualization can be done at finite temperature and on-thefly, so that the system need not to be cooled to zerotemperature and the atoms to be selected based on potential energy, where quenching can influence the system and prevent seeing the real configuration. The snapshot in Fig. 2 is when the voids have started to grow by emitting dislocations, and thus the system has already evolved somewhat through plastic flow. The broad plates 

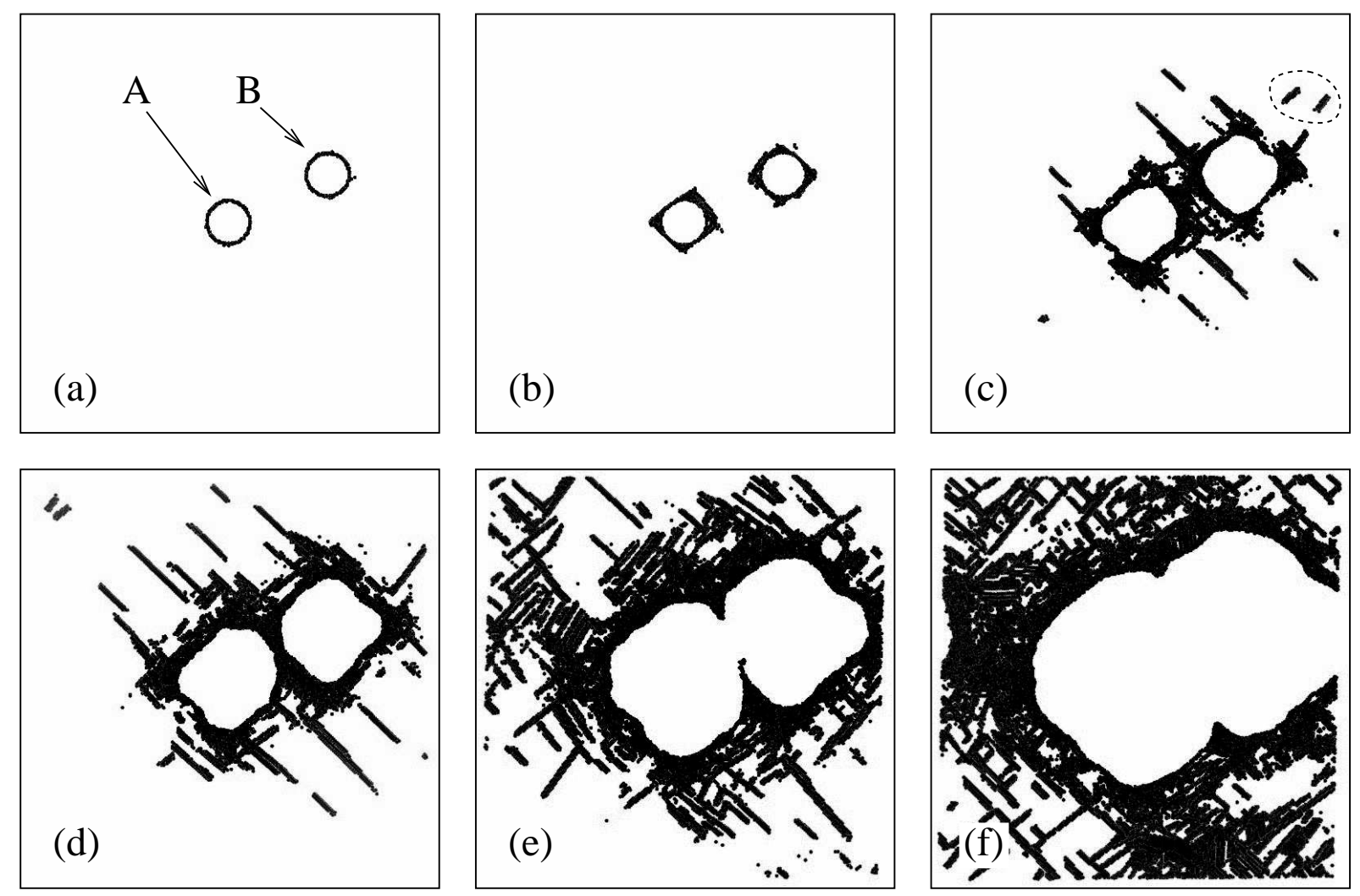

FIG. 3: Dislocation activity at six instants of time shown on one particular slice through the system in order to expose the plasticity near the void surfaces. These snapshots are from the simulation with $\dot{\varepsilon}=10^{9} /$ sec and again only those atoms in dislocation cores, stacking faults, void surfaces or other defects are shown (see text for more details and Fig. 2 for a full threedimensional figure). The dashed loop in panel (c) is drawn around a slice of a prismatic dislocation loop. The plane shown passes through the centers of both voids with normal [0.145, 0.145, -0.979]. The snapshots show the initial plasticity (a),(b), interacting plastic zones (c),(d) and the final coalescence (e),(f). The frames correspond to the following values of strain $\varepsilon$ : $1.72 \%, 2.42 \%, 3.47 \%, 3.89 \%, 4.52 \%$, and $5.21 \%$, respectively.

in the figure are stacking fault ribbons between leading and trailing partial dislocations. The first generation of dislocation loops have not yet totally formed and separated from the voids in this snapshot, but are about to do so, as can be seen as their first halves have already separated from the void surface.

Figure 3 shows a series of visualizations of the crystal defects within a slice of width $4.5 \AA$ about a plane including centers of both voids at six different instants during coalescence. These snapshots are from the same simulation as in Fig. 22 and the same criterion for showing the atoms is used. Now only a slice is shown in order to reveal the dislocation activity near the void surface as the dislocation density increases. Figure 2 is a snapshot from a state of the system between panels Fig. B(b) and (c). From the snapshots it is apparent that the deformation mechanism involves the nucleation and propagation of dislocations, accommodating the void growth, and the interaction of the dislocations ${ }^{38}$ For example, the prismatic dislocation loops punched out by the voids appear as roughly parallel line traces (due to the stacking fault ribbons) in the slice Fig. 3 (c), as verified in the full 3D configuration (cf. Fig. 2). Initially the dislocation activ- ity around each void is essentially symmetric [Figs. B(a) and (b)], as expected for independent void growth, but as the plastic fields evolve the void-void interaction is clearly evident both through interactions between the two plastic zones and bias due to the elastic fields [Fig. 3(c)]. Once the dislocation density grows sufficiently high in the ligament region between the voids [Fig. 3(d)], void B begins to grow in the direction away from void A. Next the voids coalesce [Fig. B(e)], and continue to grow as one until ultimately the void coalesces with the its periodic images [subsequent to Fig. [3(f)] so that the cavity percolates through the periodic system. The details of this final stage depend strongly on the periodic boundary conditions and will not be of interest here.

The dislocation formation is closely related to the volume evolution of the voids. The voids grow by emitting dislocation loops, driven by the reduction in the elastic energy as the increase in void volume allows the strained matrix material to relax. This relaxation can be detected from the saturation of the increasing stress in the system. All of these phenomena happen simultaneously, see Ref. 16. In Fig. 4(a) the mean (hydrostatic) stress $\sigma_{m}$ is plotted with respect to strain $\varepsilon$ 

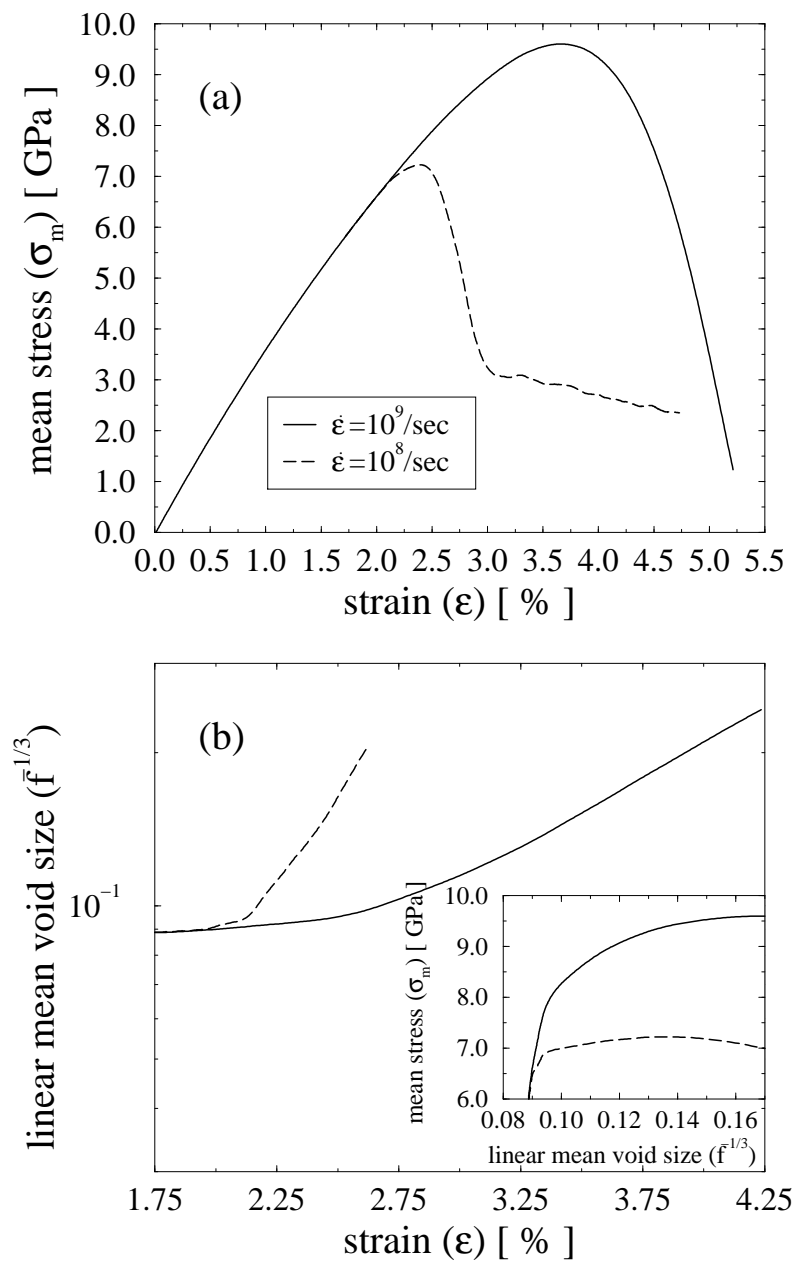

FIG. 4: (a) Mean stress $\sigma_{m}$ versus strain $\varepsilon$ from the simulations with the initial inter-void ligament distance $\operatorname{ILD}_{0}=1.81$ and the strain-rates $\dot{\varepsilon}=10^{8} / \mathrm{sec}$ (dashed line) and $10^{9} / \mathrm{sec}$ (solid line). (b) Linear mean void size, $\bar{f}^{1 / 3}$, (see the text for its definition) versus the strain from the same simulations as the data in (a). The void sizes are calculated until the coalescence of the voids. The inset shows the stress from (a) versus linear mean void size from (b). Note the strain scales are different.

(the control parameter in these simulations) for strainrates $\dot{\varepsilon}=10^{8} / \mathrm{sec}$ and $10^{9} / \mathrm{sec}$ with $\mathrm{ILD}_{0}=1.81$. The stress is calculated with the virial expression $\sigma_{m}=$ $-\frac{1}{3 V}\left(\sum_{i}\left|\vec{p}_{i}\right|^{2} / m_{i}+\sum_{i, j \mid j>i} \vec{r}_{i j} \cdot \vec{f}_{i j}\right)$, where for atoms $i$ and $j$ the $\vec{r}_{i j}$ is the relative position, $\vec{f}_{i j}$ is the force, $\vec{p}_{i}$ is the the momentum and $m_{i}$ is the mass (for a recent discussion of atomistic stress calculations see Ref. 39). Comparing Fig. 四(a) with Fig. 四(b) it is apparent that the start of the accelerated void growth due to plasticity is accompanied by, and indeed causes, the stress to plateau (at the same strain value) as the elastic dilatation is relieved. The void growth is shown in Fig. प(b) by plotting linear void size $f^{1 / 3}$, where the single void fraction is $f=V_{\text {void }} / V$ and $V$ is the instantaneous volume of
TABLE II: Critical strain $\varepsilon_{c}$ and critical linear mean void size $\bar{f}_{c}^{1 / 3}$ from Fig. [5 calculated as when the inter-void ligament distance crosses the line ILD $=0.5 d: \bar{f}_{c}^{1 / 3}=\bar{f}^{1 / 3}(\mathrm{ILD}=0.5 d)$, $\varepsilon_{c}=\varepsilon(\mathrm{ILD}=0.5 d)$.

\begin{tabular}{lccc}
\hline \hline $\mathrm{ILD}_{0}$ & $\dot{\varepsilon}\left(\mathrm{sec}^{-1}\right)$ & $\bar{f}_{c}^{1 / 3}$ & $\varepsilon_{c}(\%)$ \\
\hline 1.81 & $10^{8}$ & 0.153 & 2.46 \\
1.81 & $10^{9}$ & 0.150 & 3.48 \\
1.50 & $10^{9}$ & 0.132 & 3.22 \\
1.20 & $10^{9}$ & 0.121 & 3.06 \\
1.00 & $10^{9}$ & 0.106 & 2.74 \\
\hline \hline
\end{tabular}

the box at time $t$. The technique for calculating the void volume $V_{\text {void }}$ is described in Ref. 16. The void growth, stress saturation and even the void coalescence take place at significantly smaller strains for slower strain-rates, 16 as can be seen from the figures, too. Therefore we conclude that a natural way to plot quantities from different strain-rate simulations in the same figure is to plot them versus linear void size, $f^{1 / 3}$. Plotting versus $f^{1 / 3}$ (a derived quantity) is preferred to plotting versus the strain because it reduces strain-rate effects. Thus by choosing $f^{1 / 3}$ from Fig. 廿(b) as the reference quantity, the start of the deviation from the elastic behavior can be synchronized for the different strain-rates, see an example for the mean stress from the inset of Fig. 四 (b), where the mean stress starts to saturate for both of the strain-rates at $\bar{f}^{1 / 3} \simeq 0.09$. In the two-void case we have chosen to use the mean void fraction $\bar{f}$, which is calculated as the average void fraction of the two voids.

Throughout the remainder of the Article we will typically use the linear mean void size $\bar{f}^{1 / 3}$ as the reference quantity. The initial linear mean void fraction at ambient pressure in these simulations is $\bar{f}_{0}^{1 / 3} \simeq\left(6.0 \times 10^{-4}\right)^{1 / 3}=$ 0.084 . The initial mean void size $\bar{f}=6.0 \times 10^{-4}$ is somewhat larger than the value one gets from $\frac{4}{3} \pi\left(r_{0} / L\right)^{3}=$ $5.2 \times 10^{-4}$. This potential source of confusion arises for two reasons: first, atoms that have their centers within the radius $r_{0}$ from the void center are removed for creating the void. On the other hand, when the void volume is calculated the surface of the void is defined based on the centers of the remaining surface atoms. Second, the void surface relaxes somewhat after the void is formed. For more discussion of the void volume calculation, see Ref. 16. For reference, the mean linear void size in Fig. 2 is $\bar{f}^{1 / 3}=0.111$, and in the first four snapshots of Fig. 3 $\bar{f}^{1 / 3}=0.089,0.094,0.149$, and 0.195 , respectively. After coalescence $\bar{f}$ is not measured. 

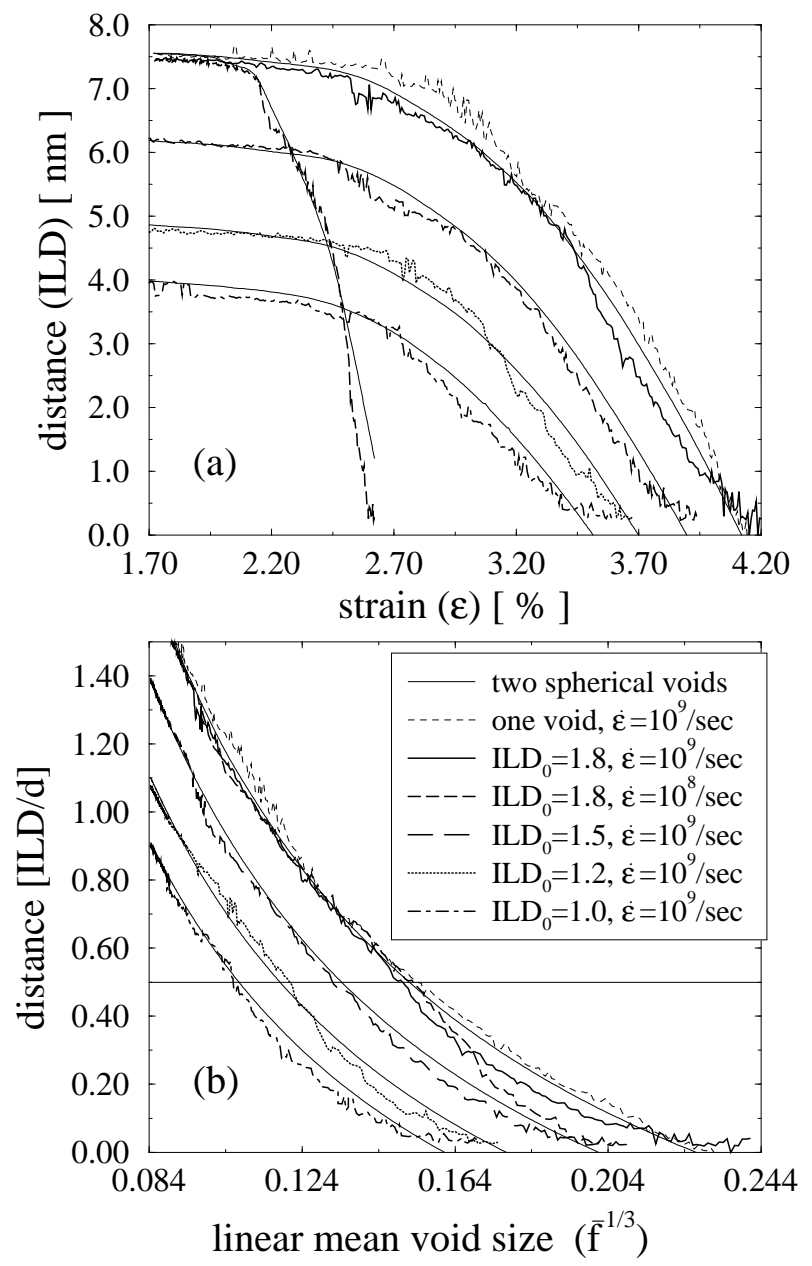

FIG. 5: Evolution of the ILD and the critical ILD. (a) Dynamical ILD, the distance between the surfaces of the voids along the line connecting the original center positions for various initial $\mathrm{ILD}_{0}=1.81,1.50,1.20,1.00$, plotted versus strain. For $\operatorname{ILD}_{0}=1.81$ the thick solid line and thick medium dashed line denote $\dot{\varepsilon}=10^{9} / \mathrm{sec}$ and $10^{8} / \mathrm{sec}$, respectively. The thin solid lines show the hypothetical ILD for spherical voids with the same $\mathrm{ILD}_{0}$ impinging freely on each other (see text). The short dashed line shows the hypothetical ILD computed by duplicating a single void (in the same box size as the two void simulations) at fixed centers (here the duplication is to the position with $\operatorname{ILD}_{0}=1.81$ ). (b) The same as in (a), but now plotted versus the linear average void size, $\bar{f}^{1 / 3}$ and the distances are given in the units of the current average diameter $d$ of the voids, calculated from their volumes assuming that they are spherical (see text). The horizontal line is at $\mathrm{ILD}=0.5$ diameters, the value we identify as the $\mathrm{ILD}_{c}$. (Panel (b) is after Ref. 27.)

\section{THE INFLUENCE OF THE NEIGHBORING VOID}

\section{A. Distance Measurements of the Voids}

Figure 3 offered several visual indications of the interaction between voids. Clearly, the separation between the void surfaces (the ILD) serves as something akin to a reaction coordinate for the coalescence: the voids coalesce when it goes to zero. Other indications include the displacement of the center of a void as it grows preferentially toward the neighboring void and the change in the void growth rate as the voids interact. In this section we now quantify two of these effects, the evolution of the ILD and the void center movement, in order to analyze the coalescence. In Section IVB we study the void shape evolution, and the void growth rate is studied in Section $\nabla$

In Figs. 15 (a) and (b) the dynamic evolution of the ILD has been plotted for strain-rates $\dot{\varepsilon}=10^{8} / \mathrm{sec}$ and $10^{9} / \mathrm{sec}$ and for various initial closest surface-to-surface distances between the voids $\mathrm{ILD}_{0}$. (In Figs. 24 the data from the case $\mathrm{ILD}_{0} \simeq 1.81$ were shown.) The dynamic ILD has been derived as the separation distance of the two surface atoms from the two voids that are closest to the line connecting the original centers of the voids. The raw data of the ILD have been plotted in Fig. 5 (a) and in Fig. 5(b) scaled data are shown. For the horizontal axis of Fig. [5), the linear mean void size $\bar{f}^{1 / 3}$ has been used in order to collapse data from different strain-rates $\dot{\varepsilon}=10^{8} / \mathrm{sec}$ and $10^{9} / \mathrm{sec}$ in the same figure. For the vertical axis the inter-void ligament distance has been divided by the current diameter of a void, $d$ (note, that it is not the initial value $d_{0}=2 r_{0}$ ). The diameter $d$ is calculated from the void volume assuming a spherical shape (the formula is given below). This scaling is motivated by the ansatz that the void diameter sets the length scale for the system, and hence the relevant distance between the voids is not the pure distance, but its ratio with the diameters of the voids. The void diameter affects both the elastic and plastic fields around the void. Initially the void separation distance decreases essentially smoothly until plasticity begins, eventually reaching zero. A transition occurs when the ILD starts to decrease noticeably faster than the free impingement line (the thin solid lines calculated from two independent spherical voids) indicating void interactions at the onset of coalescence. The thin solid lines are derived by calculating from the same simulations the average sizes of the two voids $\bar{V}_{\text {void }}$ and deriving the diameter as $d=2\left(\frac{3}{4 \pi} \bar{V}_{\text {void }}\right)^{1 / 3}$. Then the free impingement curve is given by ILD free $=\left(\mathrm{ILD}_{0}+d_{0}\right)(1+t \dot{\varepsilon})-d$. The accelerated ILD decrease associated with transition to coalescence takes place when the ILD reaches approximately one half: $\mathrm{ILD}_{c}=0.5 \pm 0.1$ diameter or one radius, independently of $\mathrm{ILD}_{0}$ or the strain-rate. Also note that a curve derived from a single void growth is provided to estimate the contribution of uncorrelated faceting effects (the "one void" curve at $\mathrm{ILD}_{0}=1.81$ ), and these effects are seen to be relatively small. They are most noticeable near the start of void growth where there is a relatively large upward fluctuation in the "one void" curve. The critical ILD of one radius is much lower than the Brown-Embury estimate, and it corresponds to a strain of $3.48 \%\left(\bar{f}^{1 / 3} \simeq 0.15\right)$ for $\mathrm{ILD}_{0}=1.81$ at $\dot{\varepsilon}=10^{9} / \mathrm{sec}$, 

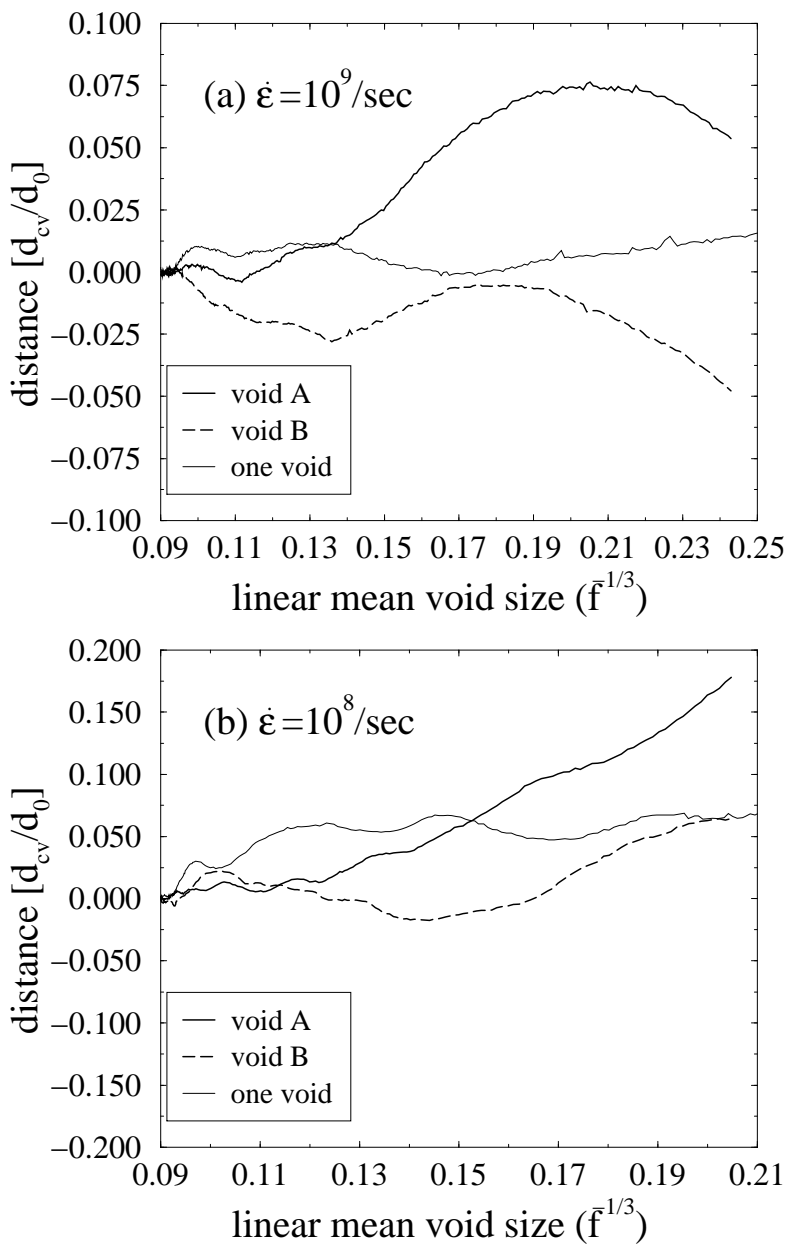

FIG. 6: Void center-of-mass displacement. The distance $d_{c v}$ from the original center of void to the instantaneous void center, projected onto the line connecting the original void centers, is plotted versus the average void size to the point of coalescence $(\mathrm{ILD} \simeq 0)$. The sign of the displacement $d_{c v}$ is positive for movement toward the other void. Solid and long dashed lines are for voids $\mathrm{A}$ and $\mathrm{B}$, respectively. Strain-rates are $\dot{\varepsilon}=10^{9} / \mathrm{sec}$ in (a) and $\dot{\varepsilon}=10^{8} / \mathrm{sec}$ in (b). The thin solid line is for a single void in the same size of the box and with the same radius and strain-rate projected to the same line. Here the distance $d_{c v}$ is given in the units of the original void diameter $d_{0} . \operatorname{ILD}_{0}=1.81$ in both (a) and (b).

close to frame (c) of Fig. 3] The values for critical strain and linear mean void size when ILD $=0.5 d$, derived from Fig. 5) (b), are tabulated for the simulated systems in Table III In the very final stages the ligament is drawn under biaxial stress, and the flow switches from radial material transport to tangential transport as the mechanism switches from loop punching to drawing. This transition is visible in Figs. [5 (a) and (b) as slowing down of the reduction of ILD. At this point, the material is highly defective but it remains ductile. There is no abrupt fracture, as might be expected at larger length scales. Here the final coalescence involves an extended drawing and thinning of the ligament until rupture.
Another measure of void interactions is whether the voids grow preferentially toward their neighbor. This effect is quantified in Fig. [6 which shows the movement of the center of mass of the void surface for the voids shown in Fig. [3 $\left(\mathrm{ILD}_{0}=1.81\right)$. We use here the center of mass of the void surface as the definition for calculations of the void center. The void surface has been derived by Voronoi triangulation based on the center points of the surface atoms (the same is done when the volume of the void is calculated). See Ref. 16 for the details of the void surface derivation. The distance

$$
d_{c v}=\left[\left(x^{\prime}-x_{c v}^{0}\right)^{2}+\left(y^{\prime}-y_{c v}^{0}\right)^{2}+\left(z^{\prime}-z_{c v}^{0}\right)^{2}\right]^{1 / 2}
$$

is calculated between the original center of mass of the void surface $\left[x_{c v}^{0}, y_{c v}^{0}, z_{c v}^{0}\right]$ and the projection $\left[x^{\prime}, y^{\prime}, z^{\prime}\right]$ of the current center of void $\left[x_{c v}, y_{c v}, z_{c v}\right]$, where the projection is onto the line connecting the original void centers. The sign is positive if the void center has moved toward the other void and negative in the opposite case.

Let us look first at the case with $\dot{\varepsilon}=10^{9} / \mathrm{sec}$, Fig. [6)(a). After the void growth starts, the center of void A initially moves only slightly, but at about $\bar{f}^{1 / 3}=0.15$ (ILD $=0.5 d$ in Fig. [5 (b) and $\bar{f}_{c}^{1 / 3}$ in Table direction of the other void as the void growth becomes biased toward its neighbor. Just before coalescence the center of void A begins to move away from void B, as the growth is biased in the opposite direction. During this sequence, void B initially grows away from void A, then roughly in unison with void $\mathrm{A}\left(\bar{f}^{1 / 3}=\bar{f}_{c}^{1 / 3}=0.15\right)$ it begins to grow toward its neighbor, and before coalescence it too switches to growth away from the proximal void. This retrograde growth happens at the same point (after $\left.\bar{f}^{1 / 3}=0.19\right)$ as the decrease of the ILD begins to slow down in Fig. [5(b) [see also the snapshot in Fig.[3(d)]. The same phenomenon-first slow movement or repulsion from the void; then growth toward the nearby void at about $\bar{f}^{1 / 3}=0.15\left(\bar{f}_{c}^{1 / 3}\right)$-holds in the $\dot{\varepsilon}=10^{8} /$ sec case, too, Fig. [6 b). However, the retrograde growth phenomenon is less pronounced in the $\dot{\varepsilon}=10^{8} / \mathrm{sec}$ case, as is the slowing down of the decrease of the ILD in Fig. (5). Indeed, only the growth toward the neighboring void is well above the noise in the $\dot{\varepsilon}=10^{8} / \mathrm{sec}$ case. As a reference the movement of the center of a single void (in same box size) projected to the same line is plotted for both strain-rates, too. Comparing the single void case with the interacting voids with the same strain-rates, one sees that the maximum distance the centers of the interacting voids have moved is 2.5 to five times larger than the nanoscale random walk of the single void center, except for the void B in Fig. 6 (b). The noise is these curves appears to be dependent on the history, reflecting the nature of the plastic deformation processes involved. It is difficult to quantify the level of noise in such a nonMarkov process, but it should be clear that the movement of the void center, especially the movement toward the neighboring void, is statistically significant and not just due to fluctuations at the void surface. 


\section{B. Shape Evolution of the Voids}

The presence of a nearby void not only affects the position of the void but its shape, as well. The shape can be quantified by calculating multipole moments of spherical harmonics $Q_{l m} \equiv \frac{1}{\bar{r}^{2}} \int Y_{l m}(\theta, \phi) r^{2}(\theta, \phi) d \Omega$, see Refs. 16 and 40. Under fully triaxial expansion the void tends to an octahedral shape because of the index of the active glide planes in FCC crystals, the anisotropic elastic constants, and anisotropic surface energies. See also Ref. 41 for a study of void shapes in FCC crystals. On the other hand, under uniaxial expansion the voids are of predominantly ellipsoidal shapes aligned along the preferred axis, and they make a transition from a prolate to an oblate shape 16 References 4243,44 have also considered oblate void shapes under uniaxial loading through continuum modeling. One may enquire whether the presence of a nearby void causes evolution to an ellipsoidal shape, see e.g. void A in Fig. 3(c). Ellipsoidal shapes can be quantified with quadrupole moments:

$$
\begin{aligned}
Q_{20} & =\frac{1}{4} \sqrt{\frac{5}{\pi}} \frac{1}{\bar{r}^{2}} \int 3 z^{2}-r^{2} d \Omega, \\
\operatorname{Re} Q_{21} & =-\frac{1}{2} \sqrt{\frac{15}{2 \pi}} \frac{1}{\bar{r}^{2}} \int x z d \Omega, \\
\operatorname{Im} Q_{21} & =-\frac{1}{2} \sqrt{\frac{15}{2 \pi}} \frac{1}{\bar{r}^{2}} \int y z d \Omega, \\
\operatorname{Re} Q_{22} & =\frac{1}{4} \sqrt{\frac{15}{2 \pi}} \frac{1}{\bar{r}^{2}} \int x^{2}-y^{2} d \Omega, \\
\operatorname{Im} Q_{22} & =\frac{1}{2} \sqrt{\frac{15}{2 \pi}} \frac{1}{\bar{r}^{2}} \int x y d \Omega,
\end{aligned}
$$

where $\bar{r}^{2}=\frac{1}{4 \pi} \int r^{2}(\theta, \phi) d \Omega$ is averaged over the surface of the void. In calculating the quadrupole moments the origin of the coordinates is taken to be the center of the void. In Eq. (2) the quadrupole moments are calculated with $\hat{z}$ as the preferred axis, whereas the physically preferred axis is the line connecting the void centers. Thus, we transform the moments to the more natural coordinates using $D$-matrices ${ }^{45}$ :

$$
\begin{aligned}
Q_{l m}\left(\theta^{\prime}, \phi^{\prime}\right) & =\sum_{m^{\prime}=-l}^{l} D_{m^{\prime} m}^{l}(\alpha, \beta) Q_{l m^{\prime}}(\theta, \phi), \\
D_{m^{\prime} m}^{l}(\alpha, \beta) & =e^{-i m^{\prime} \alpha} d_{m^{\prime} m}^{l}(\beta),
\end{aligned}
$$

where the Euler angle $\alpha$ defines the rotation between coordinates axes in $(x y)$ plane (corresponding angle $\phi$ ); and the Euler angle $\beta$ describes the rotation in $z$-axis (corresponding angle $\theta) . d_{m m^{\prime}}^{l}(\beta)$ can be found from tables ${ }^{46}$ The quadrupole moments are plotted for the voids $\mathrm{A}$ and B from the simulation with $\mathrm{ILD}_{0}=1.00$ in Figs. I7(a) and (b), respectively. One sees from Fig. 囵(a) that void A becomes markedly elliptical in the direction of the other void, as represented by $Q_{20}$, when $\bar{f}^{1 / 3} \simeq \bar{f}_{c}^{1 / 3} \simeq 0.106$ (from Table II). The quadrupole data from an identical simulation but with only one void are plotted in Fig. 7(c) as a control, and a smaller variation in the quadrupole moment is observed simply due to fluctuations in the atomistic growth. Other cases with larger $\mathrm{ILD}_{0}$ look about the same as Figs. [7(a) and (b) although the trend and especially the transition point may not be as clear. This variation may be due to the elastic
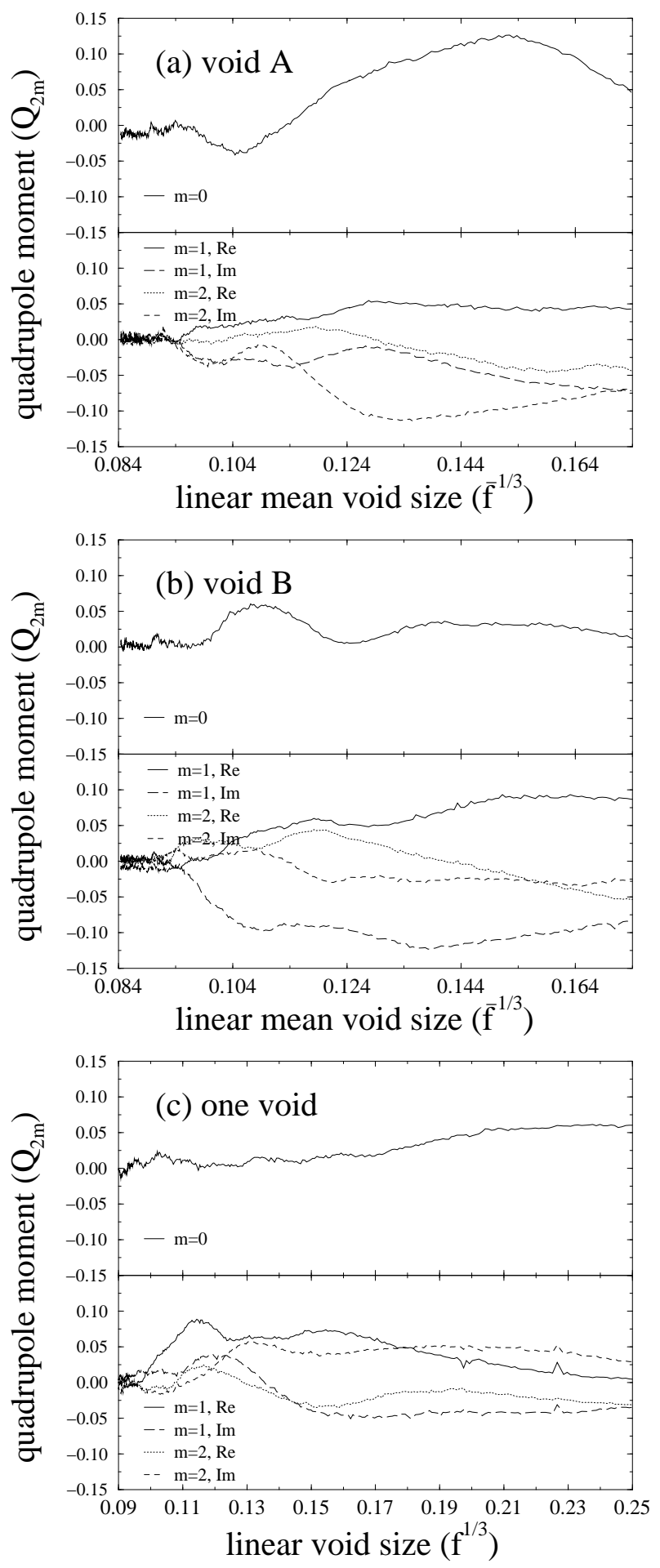

FIG. 7: (a) Quadrupole moments (2) of the surface of void A from the start of the simulation until coalescence. The coordinate axes have been rotated using Eq. (3) so that the $z$-axis is aligned with the center of void B. The initial intervoid ligament distance is $\mathrm{ILD}_{0}=1.00$ and the strain-rate is $\dot{\varepsilon}=10^{9} /$ sec. (b) Quadrupole moments for the void B, located at $[0.1778,0.0829,0.0387] L$ from void A. Now Eq. (3) has been used so that the positive $z$-axis is toward the void A. (c) Quadrupole moments (not rotated) for a single void in a simulation with same box size as in (a) and (b) and the strain-rate $\dot{\varepsilon}=10^{9} / \mathrm{sec}$. 
and plastic interactions causing random shape evolution longer before the critical $\operatorname{ILD}_{c}(\mathrm{ILD}=0.5 d)$ is reached in cases when the voids are initially separated further from each other (larger ILD $\mathrm{IL}_{0}$ ). See Fig. 7( (c) for the single void case as an example of the random shape evolution. There $\bar{f}^{1 / 3}=0.09$ is when the dislocation driven void growth starts, see inset of Fig. 4(b). Thus, in larger ILD IL cases the random shape evolution suppresses the transition to the shape evolution due to the other void.

\section{VOID VOLUME EVOLUTION UNDER THE INFLUENCE OF THE SECOND VOID}

In Ref. 27, we briefly discussed how void growth after the onset of interaction but prior to coalescence is different from void growth for an isolated void. We address this further here. How does the correlated growth differ from the exponential growth of an isolated void? ${ }^{12.16}$

\section{A. A Pair of Voids}

We first examine the volume evolution for the same two-void simulations described above. Figure 8/a) shows the void fraction, $f=V_{\text {void }} / V$, for the void A (also in the case of a single void in the same box size) with respect to the strain before coalescence. As can be seen from the figure the void grows as $\exp (200 \epsilon)$, at least for the larger $\mathrm{ILD}_{0}$ 's and for the single void in the box. In Fig. 8(b) we have factored out the asymptotic growth rate from $f$ in order to emphasize the differences between the curves and plotted versus linear void size $f^{1 / 3}$. The void growth data for $\mathrm{ILD}_{0}=4.62$ and 1.81 coincide with the single void curve. The void growth rate with smaller $\mathrm{ILD}_{0}$ 's reach their asymptotic growth rate earlier. In the figure we have drawn as circles the void size values, where the dynamic ILD's cross the line ILD $=0.5 d$ in Fig. 5(b). As can be seen from figure, there is no significant change in the void volume behavior when the voids start to interact. Therefore we conclude that the void growth rate is not affected by the interaction between the voids: thus the interaction cannot be detected through the growth rate. The key factor for the void growth rate is the rate at which the dislocations separate from the void, and it appears to remain unchanged in the vicinity of the second void. However, the location at the void surface from where the dislocation loops separate is affected by the interaction with another void, as seen in Section IV in the accelerated reduction of ILD, in the movement of void center and in the shapes of the voids.

\section{B. A Single Void Interacting with its Periodic Images}

We have also performed a series of simulations of a single void with fixed initial radius size $r_{0}$ in various initial
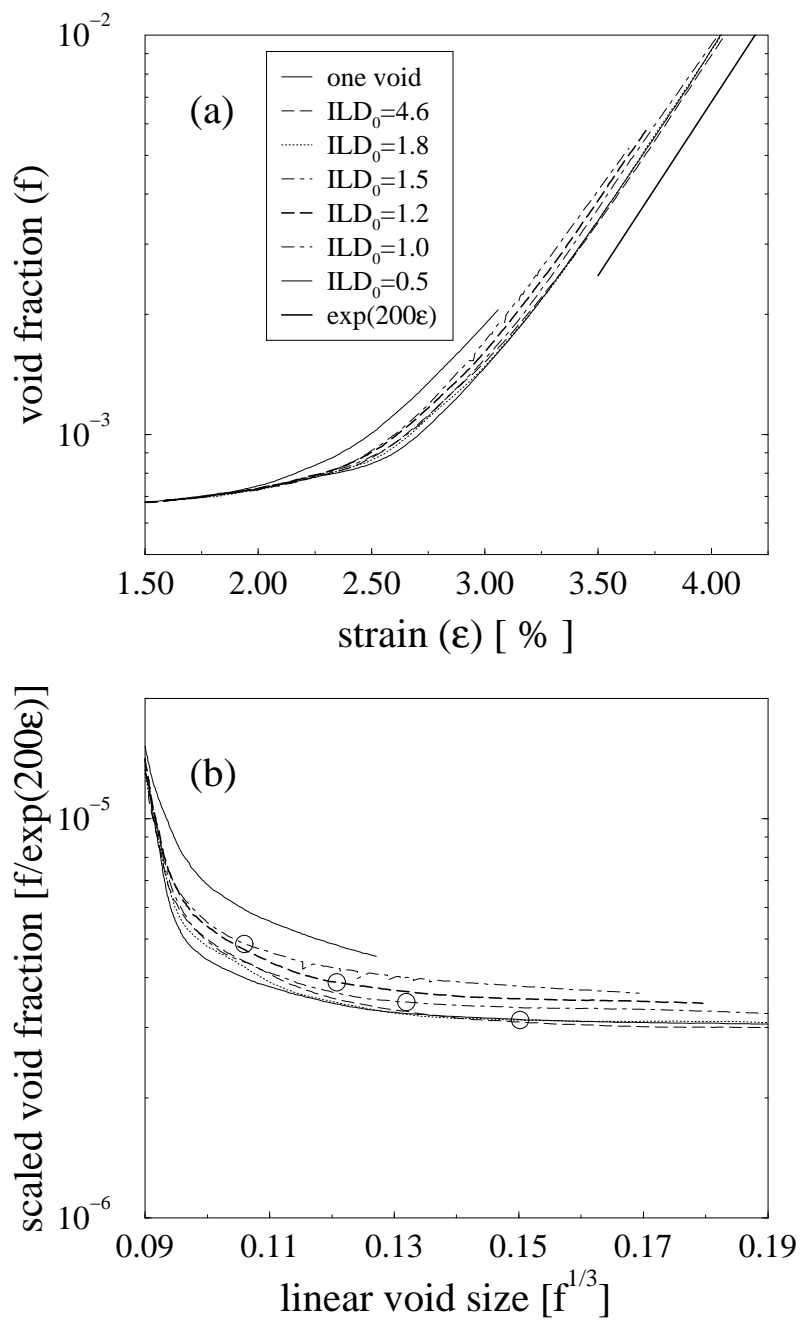

FIG. 8: (a) Growth of the void A until coalescence presented by void fraction $f=V_{\text {void }} / V$ versus strain for $\mathrm{ILD}_{0}=0.50$, $1.00,1.20,1.50,1.81$, and 4.62 diameters as well as in a single void case (in the same box size) at $\dot{\varepsilon}=10^{9} / \mathrm{sec}$. The asymptotic behavior (before finite size effects) is exponential growth with $\exp (200 \epsilon)$ as seen from the line drawn as a guide to the eye. (b) The void size $f$ presented in (a) has been scaled with the exponential and plotted versus linear void size $f^{1 / 3}$. The circles point where the dynamical ILD's cross the horizontal line ILD $=0.5 d$ in Fig. 5 (b).

box sizes $V_{0}=L^{3}$ in order to find the coalescence process of the void with its (six) periodic image(s), similar to the manner in which some continuum calculations of coalescence have been done (cf. Refs. 9 and 43 ). The details of the different box sizes are reported in Table III Figure 9 shows the data from this series of simulations. There we have scaled the void fraction $f=V_{\text {void }} / V$ with the exponential, $\exp (200 \epsilon)$ (as in Fig. 8) as well as $\left(r_{0} / L\right)^{3}$ in order to take into account different initial volumes $V_{0}=L^{3}$ of the box. Also $\left(r_{0} / L\right)$ scaling is applied to the linear void $f^{1 / 3}$ (horizontal axis). From the figure one concludes that the behavior is opposite to the results from the case of an isolated pair of voids, Section $\overline{\mathrm{A}}$ in an important way. 
TABLE III: Sizes for simulated systems with various $r_{0} / L$ $\left(r_{0}=2.17 \mathrm{~nm}\right.$ is fixed) as the number of FCC cells, the equilibrium side length of the cube $L$ at ambient pressure and room temperature, and the number of atoms in the box after the void is formed. The shortest initial inter-void ligament distance ILD $\mathrm{IL}_{0}$ of the void with its periodic image in the units of the void diameter $d$ is reported in the last column.

\begin{tabular}{ccccc}
\hline \hline$r_{0} / L$ & FCC cells & $L$ & atoms & ILD $_{0}$ \\
\hline $1 / 3$ & $18 \times 18 \times 18$ & $6.50 \mathrm{~nm}$ & 23328 & 0.50 \\
$2 / 9$ & $27 \times 27 \times 27$ & $9.75 \mathrm{~nm}$ & 78732 & 1.25 \\
$1 / 6$ & $36 \times 36 \times 36$ & $13.0 \mathrm{~nm}$ & 186624 & 2.00 \\
$1 / 8$ & $48 \times 48 \times 48$ & $17.3 \mathrm{~nm}$ & 442368 & 3.00 \\
$1 / 10$ & $60 \times 60 \times 60$ & $21.7 \mathrm{~nm}$ & 860396 & 4.50 \\
$1 / 20$ & $120 \times 120 \times 120$ & $43.3 \mathrm{~nm}$ & 6908379 & 9.00 \\
\hline \hline
\end{tabular}

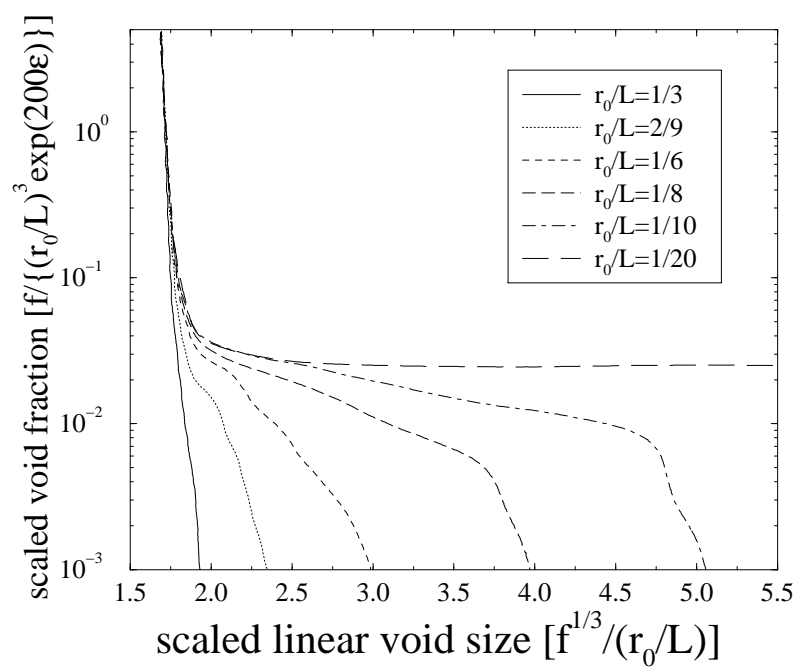

FIG. 9: Growth of a single void with varying initial box size. The initial radius of the void is kept constant $r_{0}=$ $2.17 \mathrm{~nm}$, while the initial side length of the cube is varied as $L=6.50 \mathrm{~nm}, 9.75 \mathrm{~nm}, 13.0 \mathrm{~nm}, 17.3 \mathrm{~nm}, 21.7 \mathrm{~nm}$, and $43.3 \mathrm{~nm}$. The void fraction $f$ has been divided by $\left(r_{0} / L\right)^{3}$ in order to take into account different initial volumes $V_{0}=L^{3}$ of the box. The vertical axis has been divided also with the exponential of the strain in order to show the asymptotic behavior as in Fig. 8 for the largest box size. The strain-rate is for all the simulations $\dot{\varepsilon}=10^{9} / \mathrm{sec}$.

The smaller the box size, and hence the smaller the ILD $\mathrm{IL}_{0}$ the later the void starts to grow. This is easy to understand since measured from afar the two separate voids act as one big void, and thus grow faster than a smaller void. In a single void with its periodic image that picture does not hold. For example, there is no distance at which the stress field approaches a single void stress field. The periodic image only restricts the growth of the void. At a more mechanistic level, the net effect of the array of voids is to reduce the resolved shear stress driving dislocation emission from the void surface, whereas a single nearby void enhances this resolved shear stress on some regions of the void surface. An intuitive way to understand this phenomenon is to consider that the shear stress field of a single void forces interstitial loops away from the void. This field decreases with the distance from the void as $1 / r^{3}$. At the near side of a neighboring void, this field would tend to drive interstitial loops into that void; in combination, it reduces that void's own stress field. At the far side, it adds to the other void's field, but the effect is smaller due to the greater distance. For a symmetric array, the effect is to reduce the maximum resolved shear stress across the surface and delay dislocation emission.

\section{CONCLUSIONS}

To summarize, interaction and coalescence of two voids in copper under tension have been simulated in multimillion-atom MD simulations. The effects of interactions between voids have been quantified by the increased reduction-rate of their separation, by the movement of their centers, and by their shape evolutions. The void interaction has also been visualized by detecting the dislocations moving in the system using the generalized centrosymmetry parameter. The critical inter-void ligament distance has been found to be close to one void radius, independent of the strain-rate or the initial separation distance $\mathrm{ILD}_{0}$. The onset of coalescence occurs at the point that the plastic zones surrounding the voids first interact strongly. Signatures of coalescence have been found in the dynamic ILD curves and the void center movements, as explained in detail here, including reference to the stress-strain and void volume curves. A weaker signature of the onset of coalescence has also been found in the void shape curves giving the quadrupole moment evolution. It has been demonstrated that the interaction of the voids is not reflected in the volumetric asymptotic growth rate of the voids. Finally, the coalescence process of an isolated pair of voids has been shown to be markedly different than the coalescence of a single void with its periodic images, so the latter would not provide a reliable description of coalescence in typical low-symmetry configurations.

In the future it would be interesting to study the cases with uniaxial expansion (and the various orientations of the voids with respect to the expansion direction), different sizes of the voids relative to each other, other crystal structures as body-center cubic and hexagonal lattices, and systems including larger collections of voids.

\section{Acknowledgments}

This work was performed under the auspices of the U.S. Dept. of Energy by the University of California, Lawrence Livermore National Laboratory, under contract no. W-7405-Eng-48. We would like to thank R. Becker for useful discussions. 
* Present address: Nokia Research Centre, P.O. Box 407, FIN-00045 NOKIA GROUP, Finland; Electronic address: eira@iki.fi

† Electronic address: belak@llnl.gov

‡ Corresponding author; Electronic address: robert.rudd@llnl.gov

1 High-Pressure Shock Compression of Solids II: Dynamic Fracture and Fragmentation, edited by L. Davison, D. E. Grady, and M. Shahinpoor, (Springer-Verlag, 1996).

2 T. W. Barbee Jr., L. Seaman, R. Crewdson, and D. Curran, J. of Materials 7, 393 (1972).

3 D. R. Curran, L. Seaman, and D. A. Shockey, Phys. Rep. 147, 253 (1987).

${ }^{4}$ F. A. McClintock in Metallurgical Effects at High Strain Rates, edited by R. W. Rhode, B. M. Butcher, and J. R. Holland (Plenum Press, New York, 1973).

5 J. Belak, J. Comp. Aid. Mat. Design 9, 165 (2002).

${ }^{6}$ F. A. McClintock, J. Appl. Mech. 6, 363 (1968).

7 J. R. Rice and D. M. Tracey, J. Mech. Phys. Solids 17, 201 (1969).

8 A. L. Gurson, J. Eng. Mater. and Tech. 99, 2 (1977).

9 J. Koplik and A. Needleman, Int. J. Solids Structures 24, 835 (1988); V. Tvergaard and A. Needleman, Int. J. Solids Structures 32, 1063 (1995); V. Tvergaard and A. Needleman, Int. J. Solids Structures 34, 2221 (1997); V. Tvergaard, Int. J. Solids Structures 35, 3989 (1998).

10 M. A. Zikry, Mechanics of Mater. 17, 273 (1994).

11 R. Cortes, Int. J. Solids Structures 29, 1339 (1992).

12 J. Belak, in Shock Compression of Condensed Matter, edited by Schmidt et al. (American Institute of Physics, New York, 1997), p. 211.

13 J. Belak, J. Comp. Aid. Mat. Design 5, 193 (1998).

14 J. Belak and R. Minich in Mat. Res. Soc. Symp. Proc. 539 (Materials Research Society, 1999), p. 257.

15 R. E. Rudd and J. Belak, Comp. Mat. Science, 24, 148 (2002).

16 E. T. Seppälä, J. Belak, and R. E. Rudd, Phys. Rev. B 69, 134101 (2004).

17 J. P. Bandstra and D. A. Koss, Mat. Sci. Eng. A 319-321, 490 (2001); A. B. Geltmacher, D. A. Koss, P. Matic, and M. G. Stout, Acta Mater. 44, 2201 (1996); D. M. Goto and D. A. Koss, Scripta Mater. 35, 459 (1996); P. E. Magnusen, D. J. Srolovitz, and D. A. Koss, Acta Metall. Mater. 38, 1013 (1990); P. E. Magnusen, E. M. Dubensky, and D. A. Koss, Acta Metall. 36, 1503 (1988); V. Jablokov, D. M. Goto, and D. A. Koss, Metall. and Mater. Trans. A 32, 2985 (2001).

18 D. L. Tonks, A. K. Zurek, and W. R. Thissell, in Shock Compression of Condensed Matter, edited by M. D. Furnish, N. N. Thadhani, and Y. Horie, (American Institute of Physics, New York, 2002).

19 T. Pardoen, I. Doghri, and F. Delannay, Acta Mater. 46, 541 (1998).

20 T. I. Zohdi, M. Kachanov, and I. Sevostianov, Int. J. Plasticity 18, 1649 (2002).
21 J. Benson, J. Mech. Phys. Solids 41, 1285 (1993).

${ }^{22}$ V. Tvergaard and J. W. Hutchinson, Int. J. Solids Structures 39, 3581 (2002).

23 T. Pardoen and J. W. Hutchinson, J. Mech. Phys. Solids 48, 2467 (2000).

24 V. C. Orsini and M. A. Zikry, Int. J. Plasticity 17, 1393 (2001).

25 R. Becker, J. Mech. Phys. Solids 35, 577 (1987).

26 B. P. Somerday, P. D. Pattillo II, M. F. Horstemeyer, and M. I. Baskes, Mat. Res. Soc. Symp. Proc. Vol. 578, 333 (2000).

27 E. T. Seppälä, J. Belak, and R. E. Rudd, Phys. Rev. Lett. 93, 245503 (2004).

28 J. D. Eshelby, Proc. Royal. Soc. A 252, 561 (1959).

29 L. M. Brown and J. D. Embury in Proceedings of the third International Conference on Strength of Metals and Alloys (Institute of Metals, London, 1973).

30 M. F. Horstemeyer, M. M. Matalanis, A. M. Sieber, M. L. Botos, Int. J. Plasticity 16, 979 (2000).

31 M. P. Allen and D. J. Tildesley, Computer Simulations of Liquids (Oxford University Press, Oxford, 1987).

32 D. J. Oh and R. A. Johnson, J. Mater. Res. 3, 471 (1988).

33 D. J. Oh and R. A. Johnson, in Atomistic Simulation of Materials: Beyond Pair Potentials, edited by V. Vitek and D. Srolovitz, (Plenum, New York, 1989).

34 W. G. Hoover, Phys. Rev. A 31, 1695 (1985).

35 Surface-to-surface distance between the voids refers here and in the rest of the paper to the distance between the surfaces of the voids without crossing the periodic boundaries.

36 M. Parrinello and A. Rahman, J. Appl. Phys. 52, 7182 (1981).

37 C. L. Kelchner, S. J. Plimpton, and J. C. Hamilton, Phys. Rev. B 58, 11085 (1998).

38 J. P. Hirth and J. Lothe, Theory of Dislocations, 2nd ed. (Krieger, Malabar Florida, 1982), Ch. 23.

39 J. A. Zimmerman, E. B. Webb III, J. J. Hoyt, R. E. Jones, P. A. Klein and D. J. Bammann, Modelling and Simul. Mater. Sci. Eng. 12, S319 (2004).

40 P. J. Steinhardt, D. R. Nelson, and M. Ronchetti, Phys. Rev. B 28, 784 (1983).

41 M. Hori and S. Nemat-Nasser, Mechanics of Mater. 7, 1 (1988).

42 B. Budiansky, J. W. Hutchinson, and S. Slutsky in $\mathrm{Me}$ chanics of Solids. The Rodney Hill Anniversary Volume, edited by. H. G. Hopkins and M. J. Sewell, (Pergamon Press, Oxford, 1982).

43 H. Andersson, J. Mech. Phys. Solids 25, 217 (1977).

44 P. Ponte Castañeda and M. Zaidman, J. Mech. Phys. Solids 42, 1459 (1994).

45 M. E. Rose, Elementary Theory of Angular Momentum, (John Wiley \& Sons, New York, 1957).

46 K. Hagiwara et al., Phys. Rev. D 66, 010001 (2002). 